\title{
SANS Characterization of an Anisotropic Poly(vinyl alcohol) Hydrogel with Vascular Applications
}

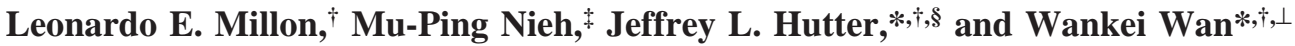 \\ Graduate Program in Biomedical Engineering, The University of Western Ontario, London, Ontario \\ N6A 5B9, Canada; Canadian Neutron Beam Center, Steacie Institute for Molecular Sciences, National \\ Research Council, Chalk River, Ontario KOJ 1JO, Canada; and Department of Physics and Astronomy \\ and Department of Chemical and Biochemical Engineering, The University of Western Ontario, \\ London, Ontario N6A 5B9, Canada
}

Received December 4, 2006; Revised Manuscript Received February 28, 2007

\begin{abstract}
Poly(vinyl alcohol) (PVA) hydrogels are formed by physical cross-linking through freeze/thaw cycles. By controlling the stress applied during the freeze/thaw process, anisotropic PVA hydrogels can be produced. An anisotropic PVA hydrogel conduit that mimics the nonlinear and anisotropic mechanical properties displayed by porcine aorta was developed. Preliminary structural characterization of isotropic and anisotropic PVA samples using small-angle neutron scattering reveals a polymer mesh cross-linked by crystallites spaced by about $18 \mathrm{~nm}$ and, most importantly, that the anisotropic properties are due to large-scale $(>100 \mathrm{~nm})$ structures alone; the geometry of the polymer mesh and crystallites remains largely unaltered. Controlling the properties of these anisotropic PVA hydrogels promises a broad range of potential applications in biomedical devices, such as coronary bypass grafts, where compliance mismatch between the implanted synthetic graft and the surrounding tissue has been identified as a major cause of failure.
\end{abstract}

\section{Introduction}

In coronary bypass graft surgery, a suitable length of the patient's saphenous vein or several types of arteries is harvested and used. However, the supply of these vessels may not be sufficient for multiple bypass or repeat operations; moreover, they tend to deteriorate due to further advancement of the patient's coronary artery disease, such as thrombi, neointimal formation, or arteriosclerosis. ${ }^{1-3}$ Polymeric materials have been used in the fabrication of synthetic cardiovascular prostheses. The most popular FDA approved polymers in use as synthetic vascular prostheses are poly(ethylene terephthalate) (PET, Dacron) and expanded polytetrafluoroethylene (ePTFE, Goretex). These synthetic grafts perform well as large diameter $(>6$ $\mathrm{mm})$ peripheral grafts with high-flow and low-resistance conditions but are not suitable for small diameter $(<4 \mathrm{~mm})$ arterial reconstructions because they are prone to thrombus induction and stenosis. Another important consideration is the mismatch in mechanical properties between the graft material and native tissue at the suturing junction. It is believed that this plays a major role in postoperative complications and ultimate graft failure ${ }^{4-6}$ Thus, there is a need to create a biomaterial with properties tuned for specific cardiovascular device applications.

Cardiovascular tissues typically consist of a large proportion of elastin and collagen, where elastin provides the initial elasticity and the collagen fibers contribute to the arterial wall tension as the vessel is progressively stretched. ${ }^{7,8}$ The aortic tissue has an anisotropic nonlinear mechanical response, with

\footnotetext{
* Corresponding authors. e-mail: wkwan@eng.uwo.ca; Ph 519-661-2111 x88440; Fax 519-850-2308. (W. W.); e-mail: jhutter@uwo.ca; Ph 519-661$2111 \times 86719$; Fax 519-661-2033 (J. L. H.).

Graduate Program in Biomedical Engineering, The University of Western Ontario.

$\stackrel{+}{\dagger}$ National Research Council.

$\S$ Department of Physics and Astronomy, The University of Western Ontario.

${ }^{\perp}$ Department of Chemical and Biochemical Engineering, The University of Western Ontario.
}

the circumferential direction having a higher elastic modulus than the axial direction..$^{9,10}$

Poly(vinyl alcohol) (PVA) is a biocompatible hydrophilic polymer that can be transformed from a solvated state to a solid hydrogel by physical cross-linking using low-temperature thermal cycling. This produces a network of crystallites linked via polymer chains and separated by solvent. ${ }^{11-14}$ The mechanical properties of PVA are similar to the characteristics of soft tissues, displaying an exponential stress-strain curve that can be altered by changing several physical cross-linking parameters. ${ }^{15,16}$ Wan et al. ${ }^{15}$ reported PVA samples that matched the circumferential direction in porcine aortic root up to $35 \%$ strain. Millon et al. ${ }^{17}$ reported a PVA-bacterial cellulose nanocomposite that was able to closely match the mechanical properties of porcine aorta in either circumferential or axial directions up to $70 \%$ strain as well as a nanocomposite with high composition of both components which fell within the range displayed by heart valve tissues. These PVA gels and PVA-based nanocomposites displayed isotropic behavior. In our latest study, we developed a novel physically cross-linked anisotropic PVA hydrogel able to closely match the anisotropy of porcine aorta in both directions (up to $65 \%$ strain) in a sheet configuration. ${ }^{18}$

The structure of PVA hydrogels has been probed by confocal microscopy, ${ }^{19}$ transmission electron microscopy (TEM), solidstate nuclear magnetic resonance $\left({ }^{13} \mathrm{C} \mathrm{NMR}\right)$ and small-angle $\mathrm{X}$-ray scattering (SAXS) ${ }^{20}$ light scattering, ${ }^{21}$ and both smalland ultrasmall-angle neutron scattering (SANS and USANS, respectively). ${ }^{22-24}$ On the basis of his study, Willcox et al. ${ }^{20}$ proposed a model for the formation of these gels whereby ice crystals formed during the low-temperature phase of the thermal cycles concentrate the polymer solution in regions between the ice crystals, promoting the formation of PVA crystallites. The crystallites act as cross-linking centers for dangling chains, which can bridge the gap between neighboring crystallites. Upon melting, the ice crystals create pores of polymer-poor solution regions embedded in the surrounding polymer-rich gel. During successive thermal cycles, ice crystals form preferentially in the 


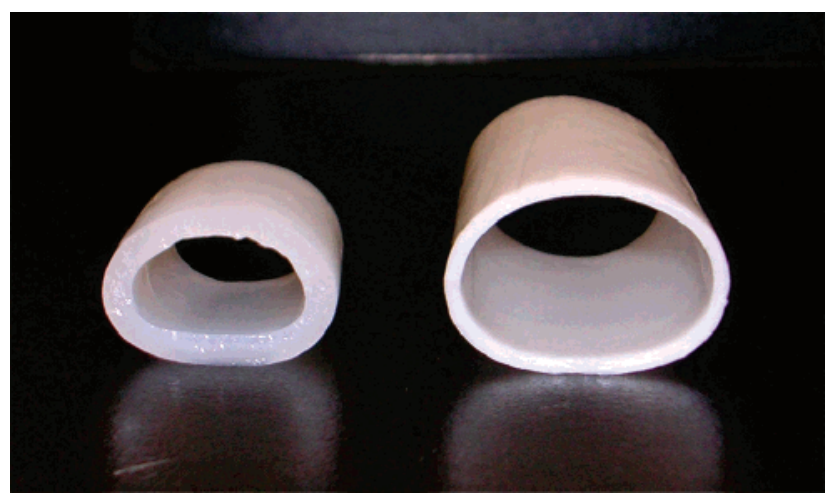

Figure 1. PVA conduit (cycle 1, left) and the $75 \%$ initially strained PVA conduit (cycle 3, right).

pores, further enhancing polymer crystallization in the gel, resulting in a stiffer material.

SANS and other techniques were used successfully by Ricciardi et al. ${ }^{25-30}$ to determine the fundamental length scales of PVA hydrogels. They proposed a model consisting of a polymer-rich mesh (the swollen amorphous phase) containing crystallites with sizes of $2-8 \mathrm{~nm}$ separated by an average distance of $20 \mathrm{~nm}$ and larger features $(>100 \mathrm{~nm})$ corresponding to polymer-poor pores. In other studies it was notable that the size of the smaller feature is nearly independent of PVA concentration and degree of thermal processing, supporting the picture of a gel whose fundamental geometry is fixed by ice crystallization in the initial thermal cycle. ${ }^{21,24}$

The purpose of this study is to demonstrate that this technique of forming anisotropic PVA samples can be used to develop an anisotropic conduit that mimics the tensile behavior of porcine aorta. Most importantly, we also address the microstructure responsible for this anisotropy by using SANS to study anisotropic PVA sheets prepared using $\mathrm{D}_{2} \mathrm{O}$ solvents.

\section{Materials and Methods}

PVA Sample Preparation. PVA (Sigma-Aldrich Canada) with a molecular weight $\left(M_{\mathrm{w}}\right)$ of 146 000-186 000 (99+\% hydrolyzed) was used in all solution preparations. The following procedures were employed to prepare distilled water samples for the anisotropic PVA conduit and $\mathrm{D}_{2} \mathrm{O}$ samples for SANS experiments.

(a) Anisotropic PVA Conduit. A 10 wt \% PVA solution in distilled water was prepared using a Pyrex flask equipped with mixing and reflux for $3 \mathrm{~h}$ at $90^{\circ} \mathrm{C}$, similar to the procedure reported previously..$^{18}$ The solution was transferred into a custom-designed aluminum tubular mold in the form of two concentric cylinders 17 $\mathrm{cm}$ long. The outer diameter (o.d.) of the inner cylinder was 16.8 $\mathrm{mm}$, and the inner diameter (i.d.) of the outer cylinder was 22.8 $\mathrm{mm}$. The mold, filled with PVA solution, was placed in a heated/ refrigerated circulator and cycled between 20 and $-20{ }^{\circ} \mathrm{C}$ once to give a cycle 1 sample, following a similar procedure to that described previously. ${ }^{18}$ This produced a conduit with an i.d. of 16.8 $\mathrm{mm}$ and a wall thickness of $3 \mathrm{~mm}$.

The cycle 1 PVA conduit was then stretched onto a larger diameter cylinder (o.d. $=29.36 \mathrm{~mm}$ ), giving a $75 \%$ initial strain. The stretched sample was cycled two more times, to obtain a cycle 3 conduit with a final thickness of $2 \mathrm{~mm}$. Figure 1 shows both the original cycle 1 conduit and the $75 \%$ strained PVA conduit cycle 3. Notice that the weaker cycle 1 PVA conduit (isotropic) is less opaque than the stiffer cycle 3 PVA (anisotropic) conduit. After cutting half of the conduit, the other half (still on the cylinder) was cycled 3 more times to obtain a cycle 6 sample for comparison to the cycle 6 sheets reported previously. ${ }^{18}$

All samples were cut into $25 \mathrm{~mm} \times 5 \mathrm{~mm}$ strips in either the circumferential or axial directions for mechanical testing (five samples for each case).

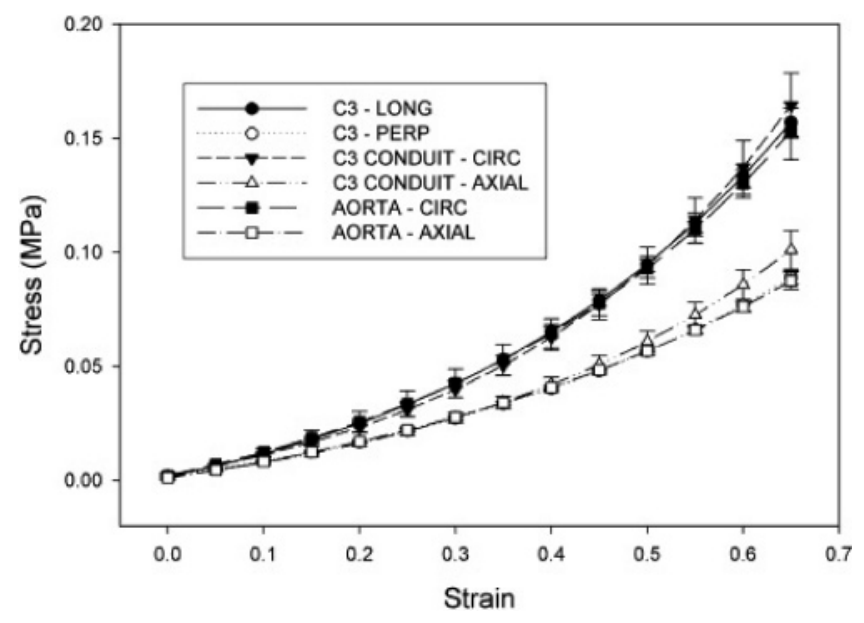

Figure 2. Stress-strain curves for the anisotropic PVA conduit $(75 \%$ initial strain, cycle 3), the anisotropic PVA sheet sample (75\% initial strain, cycle 3 ), and porcine aorta (both directions).

(b) Anisotropic PVA- $\mathrm{D}_{2} \mathrm{O}$ for SANS Experiments. Samples for neutron scattering were prepared using the same PVA (SigmaAldrich Canada), as described above. A $10 \mathrm{wt} \%$ PVA solution in $\mathrm{D}_{2} \mathrm{O}$ (Sigma-Aldrich Canada) with a 99.9 atom \% deuteration was prepared in a $250 \mathrm{~mL}$ round-bottom flask with mixing and a reflux column. The reflux column was mounted with a drying tube containing anhydrous calcium sulfate (Drierite, Sigma-Aldrich Canada) to prevent moisture from entering the reaction flask. The solution was heated for $3 \mathrm{~h}$ at $90{ }^{\circ} \mathrm{C}$ and poured into flat aluminum molds which underwent one freeze/thaw cycle. ${ }^{18}$ The cycle 1 sample ( $\sim 1.6 \mathrm{~mm}$ in thickness) was stretched and secured in a customdesigned mold at $75 \%$ initial strain, which was the same strain as for the tubular configuration. A sample not subjected to strain was also placed in the mold for use as a control. Since the stiffest material with a high degree of anisotropy was reported to be the cycle 6 sample, ${ }^{18}$ the stretched PVA- $\mathrm{D}_{2} \mathrm{O}$ sheet and control were cycled five more times to obtain cycle 6 samples.

All samples for neutron scattering were cut into $17 \mathrm{~mm} \times 17$ $\mathrm{mm}$ squares, with reference notches at the corners to indicate the direction of stretch. Anisotropic samples and isotropic controls were sent to the National Institute of Standards and Technology (NIST) in vials filled with $\mathrm{D}_{2} \mathrm{O}$. The leftover PVA- $\mathrm{D}_{2} \mathrm{O}$ samples, both anisotropic and control (three samples for each case), were cut ( 25 $\mathrm{mm} \times 5 \mathrm{~mm}$ ) in either the direction longitudinal or perpendicular to the applied stress for mechanical testing.

Mechanical Tensile Testing. The mechanical testing equipment consisted of a servo-hydraulic material testing system (INSTRON 8872) equipped with a $1 \mathrm{~kg}$ load cell (Transducer Techniques model GS-1000). The sample thickness was measured using a customdesigned Mitutoyo gauge thickness tester. Testing for all samples was carried out in a Plexiglas tank filled with distilled water kept at $37^{\circ} \mathrm{C}$. All the specimens were placed into a custom-designed grip (grip-to-grip distance of $10 \mathrm{~mm}$ ), and uniaxial tensile tests were performed at a crosshead speed of $40 \mathrm{~mm} / \mathrm{s}$ to $65 \%$ strain. All samples were preconditioned with 10 loading and unloading cycles. The load vs extension data were converted to engineering stressengineering strain, using the sample thickness and the initial gauge length after preconditioning, as reported previously. ${ }^{17,18}$

Neutron Scattering Experiments. PVA- $\mathrm{D}_{2} \mathrm{O}$ samples for neutron scattering were loaded into standard NIST demountable cells with quartz windows. Two PVA hydrogel sheets were stacked in each $4 \mathrm{~mm}$ path length cell, with the remaining volume filled with $\mathrm{D}_{2} \mathrm{O}$, for each measurement (yielding a transmission of $\sim 75 \%$ ). In the case of anisotropic samples, care was taken that both sheets had the same alignment, as determined by their reference notches.

SANS was performed on the $30 \mathrm{~m}$ NG3 SANS beamline at NIST. This instrument is suitable for examining structural features in materials ranging from roughly 1 to $500 \mathrm{~nm}$. The raw SANS intensity was collected by a 2-D detector, corrected by the 


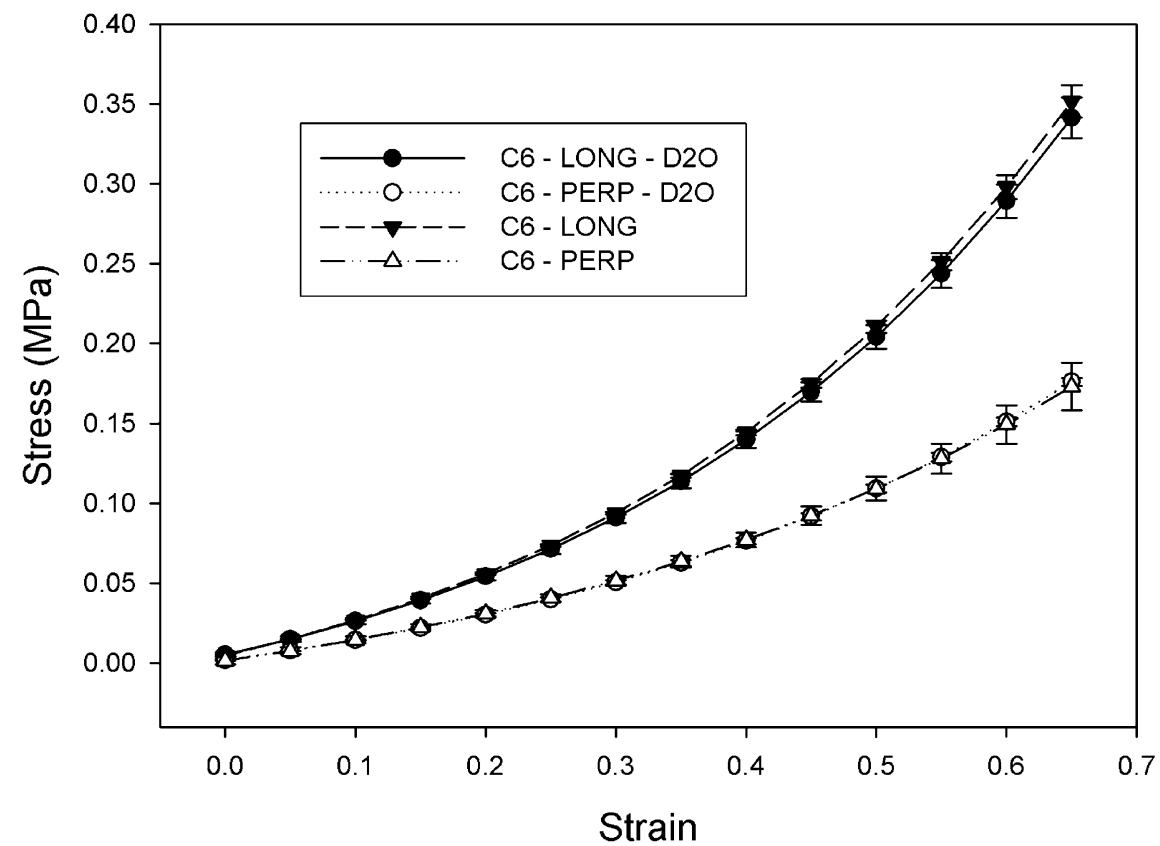

Figure 3. Stress - strain curves for the anisotropic PVA $-\mathrm{D}_{2} \mathrm{O}$ and PVA (distilled water) samples (both samples 75\% initial strain, cycle 6).

background, and normalized to the neutron flux to be put on an absolute scale. Generally, the SANS intensity is presented as a function of scattering vector, $q$, which is defined as $(4 \pi / \lambda) \sin (\theta / 2)$, where $\lambda$ and $\theta$ are the wavelength of the neutrons and the scattering angle, respectively. In order to achieve a $q$ range from 0.001 to $0.09 \AA^{-1}$ and to ensure enough overlapping domain, we set up two configurations. For the high $q$ range, a sample-detector distance (sdd) of $5 \mathrm{~m}$ and a neutron wavelength of $5.5 \AA$ were employed. For the low $q$ range, a sdd of $15.3 \mathrm{~m}$, a wavelength of $8.09 \AA$, and the focusing lens were employed.

\section{Data Analysis}

Tensile Tests. The stress-strain data for all PVA materials, isotropic or anisotropic, is nonlinear, with an increasing slope over the range tested. The stress-strain data was fit to eq 1, as reported previously: ${ }^{17,18,31}$

$$
\sigma=y_{0}+A \exp (B \epsilon)
$$

where $\sigma$ is the engineering stress, $\epsilon$ is the engineering strain, and $y_{0}, A$, and $B$ are curve-fitting parameters.

For cardiovascular tissues, the approximate average strain between diastole and systole for porcine aortic root ranges between 20 and 30\%:15,32 thus, comparisons of properties against aortic tissues are performed at $30 \%$ strain. This limit is also used for cyclic loading and unloading of the samples up to 10000 times.

The mechanical properties of different samples were compared using two-way analysis of variance (ANOVA), which is consistent with the statistical analysis performed previously. ${ }^{15,17,18}$

SANS Data Analysis. 2-D patterns of absolute scattering intensity were obtained for each sample and each $q$ range. The 2-D scattering patterns for isotropic samples exhibit a circularly symmetric pattern, while those of the anisotropic samples are distinctly elliptical, particularly at low $q$, with the longest axis representing the direction perpendicular to the strain applied during processing (as shown in the Results section).

For the isotropic sample, 1-D intensity as a function of $q$ was conveniently obtained by circularly averaging the 2-D data. For the anisotropic sample, two curves were generated, one measured longitudinal and one perpendicular, to the direction of the strain imposed during processing by restricting the circular average to sectors spanning $30^{\circ}$ and centered on the semiminor and semimajor axes of the elliptical pattern.

\section{Results}

Note on Notation: In the following figures, data from conduits (either PVA or porcine aorta) are denoted (CIRC) for circumferential samples and (AXIAL) for axial samples (along the length of the conduit). For the sheets (either PVA in distilled water or $\mathrm{D}_{2} \mathrm{O}$ ), samples cut in the direction of applied stress are denoted as (LONG) for longitudinal, and samples cut perpendicular to the applied stress are denoted (PERP). Isotropic (unstrained) samples are denoted (ISO).

Tubular PVA Conduit. Millon et al. ${ }^{18}$ reported that cycle 3 anisotropic PVA sheets (75\% initial strain) are able to closely match the stress-strain curves for porcine aorta in both circumferential and axial directions. Figure 2 shows that tubular specimens prepared under similar conditions $(75 \%$ initial circumferential strain, cycle 3) yield stress-strain curves similar to porcine aorta (both directions) and the anisotropic PVA sheets. In order to quantify the similarity in the curves, the data were compared at a strain of $30 \%$. An ANOVA test comparing the longitudinal direction of the PVA sheets, the circumferential direction of the PVA conduits, and the circumferential direction of the porcine aorta showed no statistical difference $(P>0.05)$. Similarly, the perpendicular direction (PVA sheets), axial direction (PVA conduits), and axial direction (porcine aorta) agreed to the same statistical significance. This is the first polymeric hydrogel device that matches the geometry and tensile properties of aortic tissue.

The effect of repeated cycling was assessed for the cycle 3 anisotropic PVA. Three samples underwent 1, 100, 1000, and 10000 loading and unloading cycles between 0 and $30 \%$ strain (physiological range) in the circumferential direction. The stress - strain response was unchanged after continuous loading and unloading cycles, and the stress values at $30 \%$ strain were found to be statistically the same $(P>0.05)$ for all three samples up to 10000 cycles.

Mechanical Properties of PVA- $-\mathbf{D}_{2} \mathbf{O}$. For neutron scattering experiments, it was necessary to prepare PVA hydrogel samples 
using fully deuterated water. Prior to the SANS experiments, we verified that samples thus prepared had the same mechanical behavior as the previously studied gels. Figure 3 shows stress strain curves for the anisotropic PVA $-\mathrm{D}_{2} \mathrm{O}$ and PVA (distilled water) samples ${ }^{18}$ (both samples $75 \%$ initial strain, cycle 6). It is evident that the mechanical properties of the PVA hydrogel prepared with $\mathrm{D}_{2} \mathrm{O}$ are in close agreement with the data reported for PVA samples with distilled water. At $65 \%$ strain, the ratio of the longitudinal to the perpendicular stress was 1.9, similar to the value of around 2 previously reported. ${ }^{18}$ When ANOVA was applied to the stress (at 30\% strain) in the longitudinal direction of both the PVA $-\mathrm{D}_{2} \mathrm{O}$ and PVA samples, there was no statistical difference $(P>0.05)$. Similarly, the perpendicular direction of both the PVA $-\mathrm{D}_{2} \mathrm{O}$ and PVA samples agreed to the same statistical significance.

Mechanical Properties of Aged PVA. In order to be useful as long-term medical devices, the effects of aging on the mechanical properties of PVA hydrogels must be investigated. The mechanical properties of anisotropic PVA (75\% initial strain, cycle 6) after storage in water for more than 1 year (13 months) were tested again and compared to its initial properties. When ANOVA was applied to the stress (at 65\% strain) in the longitudinal and perpendicular directions, the two sets (corresponding to initial and aged samples) were found to be statistically the same $(P>0.05)$.

SANS Results for PVA- $-\mathbf{D}_{\mathbf{2}} \mathbf{O}$. As described previously, the neutron scattering data were reduced to obtain 2-D patterns of absolute scattering intensity for each sample (isotropic and anisotropic), one for each $q$ range used. Parts a and b of Figure 4 show the 2-D patterns of absolute scattering intensity for isotropic PVA samples (cycle 6) at high $q$ and low $q$, respectively, while parts a and b of Figure 5 show similar patterns for anisotropic PVA samples (cycle 6) at high $q$ and low $q$, respectively. The anisotropic patterns yield a distinctly elliptical pattern, particularly in the low $q$ regime, indicating that its structure has been elongated along the stretching (vertical) direction.

As explained earlier, 1-D functions representing the scattering cross section as a function of $q$ were obtained for each case. The isotropic curve was obtained by circular averaging of the 2-D data, while the data from the anisotropic samples were averaged in $30^{\circ}$ sectors about the axes longitudinal and perpendicular to the direction of stretch (e.g., the green line in Figure $5 \mathrm{~b}$ indicates the perpendicular direction and the blue lines the $30^{\circ}$ sectors). Figure 6 shows $1-\mathrm{D} \log -\log$ plots of differential scattering cross section vs scattering vector for the isotropic PVA sample and both directions (longitudinal and perpendicular to the applied strain) of the anisotropic PVA. All three curves overlap nearly exactly at high $q\left(>0.06 \AA^{-1}\right)$ (the curve for the isotropic sample has been offset by a factor of 10 to avoid clutter), with differences occurring mainly at lower $q$ values.

SANS data for PVA hydrogels have been modeled previously $22,23,28,30$ with varying degrees of sophistication. In the present study, the modest goal is to compare the characteristic length scales of isotropic PVA samples (cycle 6) and the two directions of our anisotropic sample (cycle 6, 75\% initial strain).

Inspection of the data shown in Figure 6 reveals several regions: (i) a steep tail (of power law nearly -4) at low q; (ii) a plateau region near $q=0.03 \AA^{-1}$; (iii) a shoulder within which the curve rolls off to (iv) a second slope of approximately -4 at high $q$. The position of the shoulder defines the length scale of the structures responsible for the power law scattering in region iv ( -4 slope region).
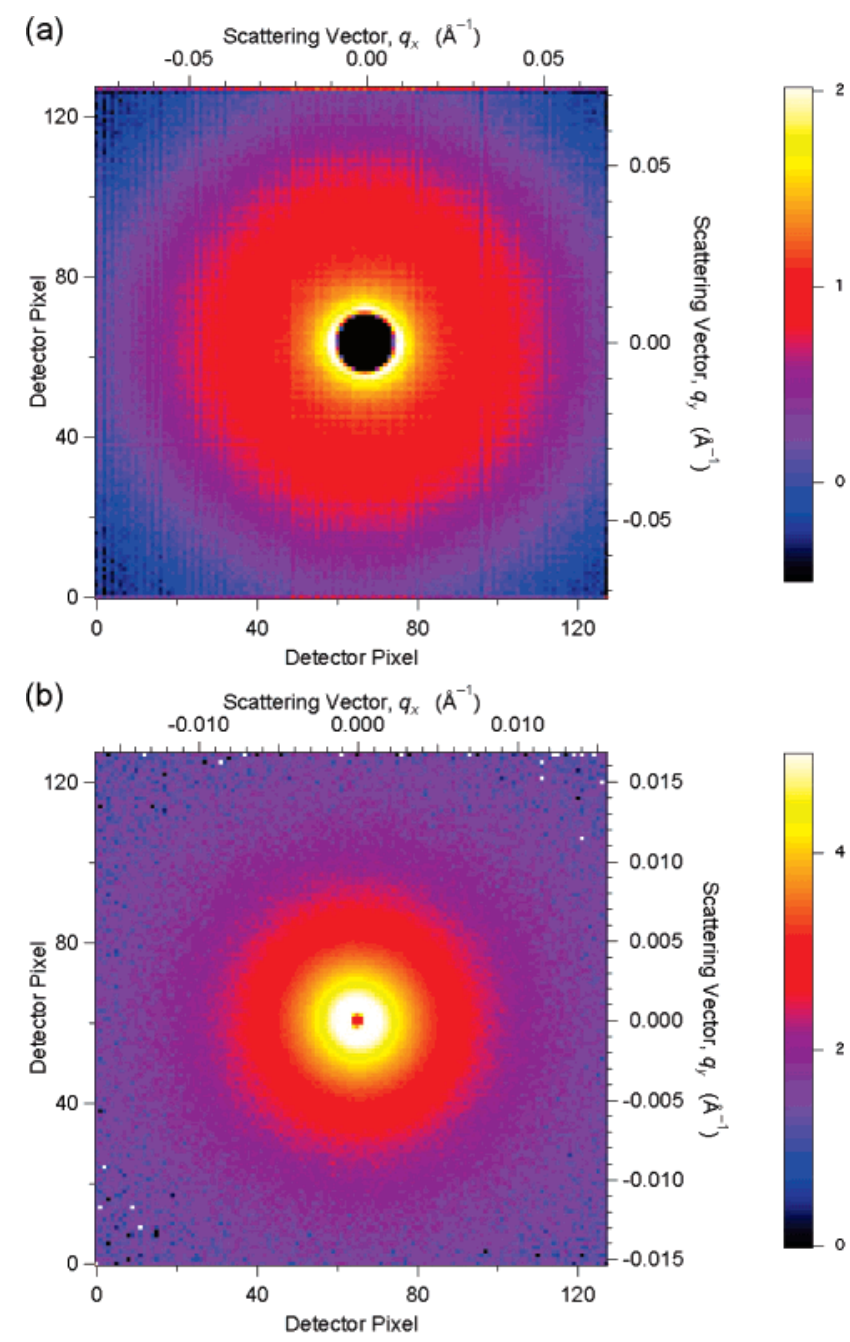

Figure 4. 2-D pattern of scattering intensity for isotropic PVA at (a) high $q$ and (b) low $q$.

The steep slope of -4 at high values of $q$ indicates scattering from sharp interfaces, the so-called Porod law, and rules out any significant contribution from free polymer, which would contribute a slope of only -2 (the Debye model). ${ }^{33}$ The simplest model to fit the plateau and $q^{-4}$ decay at the high- $q$ regime is the one-parameter Debye-Bueche $(\mathrm{D}-\mathrm{B})$ model, given by ${ }^{33}$

$$
\frac{\mathrm{d} \Sigma}{\mathrm{d} \Omega}=\frac{A}{\left[1+(\xi q)^{2}\right]^{2}}
$$

which is the expected dependence on $q$ for scattering from randomly distributed domains in a two-phase system of characteristic size $\xi$ (correlation length). The coefficient $A$ contains information about the scattering contrast between domains as well as the number of domains. Note that at high $q$ (i.e., $q \gg$ $1 / \xi)$, the scattering decays as $q^{-4}$, as predicted by the Porod law.

We find, however, that the $\mathrm{D}-\mathrm{B}$ model is unable to match the narrow shoulder present in the data and does not roll off to the experimental slope of -4 quickly enough. This implies a greater degree of order (i.e., a narrower size distribution) than assumed by the $\mathrm{D}-\mathrm{B}$ model. A better match is obtained with the Teubner-Strey (TS) model which describes a quasi-periodic arrangement of domains of average size $d$ that are correlated over a distance $\xi$, and is commonly used to model scattering 
(a)

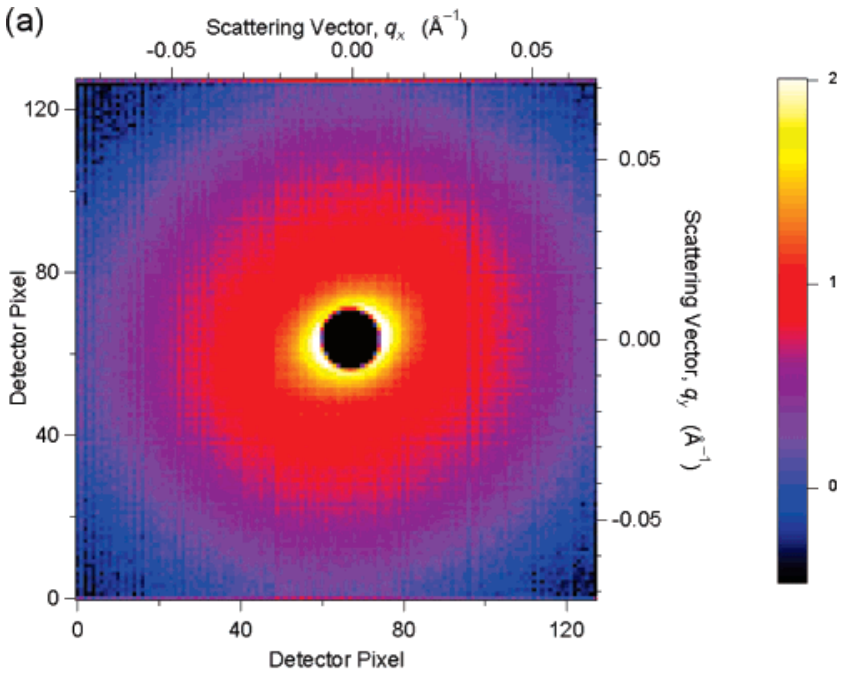

(b)
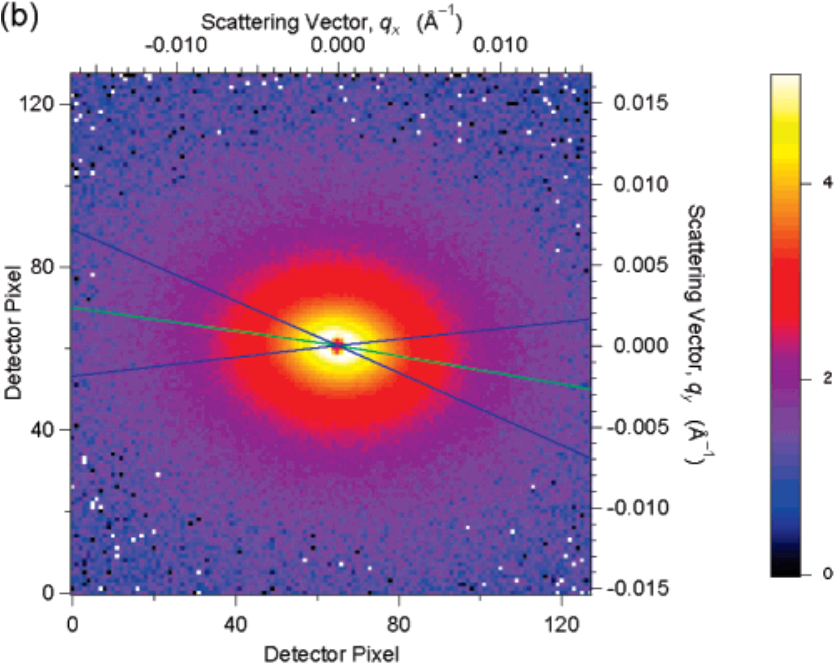

Figure 5. 2-D pattern of scattering intensity for anisotropic PVA at (a) high $q$ and (b) low $q$ (the green line indicates the perpendicular direction, and the blue lines denote the $30^{\circ}$ sectors used to estimate the differential scattering cross section in the perpendicular direction).

from microemulsions. ${ }^{34}$ The Teubner-Strey model predicts a scattering intensity of the form

$$
\frac{\mathrm{d} \Sigma}{\mathrm{d} \Omega}=\frac{1}{a_{2}+c_{1} q^{2}+c_{2} q^{4}}
$$

The fit parameters are related to a quasi-periodic repeat distance

$$
(2 \pi / d)^{2}=\frac{1}{2} \sqrt{\frac{a_{2}}{c_{2}}}-\frac{c_{1}}{4 c_{2}}
$$

and correlation length

$$
1 / \xi^{2}=\frac{1}{2} \sqrt{\frac{a_{2}}{c_{2}}}+\frac{c_{1}}{4 c_{2}}
$$

The scattering in the low- $q$ region (i) indicates structures much larger than those responsible for the high- $q$ shoulder. However, because the data do not level off, even at the lowest values of $q$ probed, this length scale cannot be determined confidently nor can a valid choice be made between alternative models for this regime. For these reasons, we simply fit the data at low $q$ to a four-parameter empirical model due to
Beaucage, ${ }^{35}$ which has been used to approximate the scattering from a wide variety of structures:

$$
\frac{\mathrm{d} \Sigma}{\mathrm{d} \Omega}=G \exp \left(-q^{2} R_{\mathrm{g}}{ }^{2} / 3\right)+B\left[\operatorname{erf}\left(q R_{\mathrm{g}} / \sqrt{6}\right)\right]^{3 P} / q^{3}
$$

The fit parameter $R_{\mathrm{g}}$ characterizes the size of the structures responsible for the scattering, and the power law exponent $P$ is characteristic of their fractal dimension. The scaling parameter $G$ gives the limiting scattering at $q=0$.

The three 1-D data sets of differential scattering cross section $\mathrm{d} \Sigma / \mathrm{d} \Omega$ vs scattering vector $q$ were fit to a sum of the TeubnerStrey and Beaucage forms. The relevant length scales thus estimated are shown in Table 1. Because of their dependence on model details (e.g., the form assumed for low $q$ and imposed constraints), no uncertainties in the fit parameters are provided. In Figure 6, the contributions from the Beaucage (dashed curve) and Teubner-Strey (dotted curve) terms are shown for the scattering from the anisotropic sample longitudinal to the strain direction.

\section{Discussion}

Among the many properties a material must possess in order to be considered for medical device fabrication, mechanical properties have been highlighted in numerous applications. In cardiovascular devices, compliance mismatch between the relatively inelastic synthetic grafts that are currently available and the elastic native artery is believed to contribute to intimal hyperplasia $(\mathrm{IH})$ and their ultimate failure. A good replacement should retain circumferential and axial compliance and pulsatility, allowing sutures to hold under both types of tension. ${ }^{1,4}$ Since most tissues display anisotropic mechanical properties, it is important to take this into account when designing a biomaterial for specific applications. ${ }^{8}$ The dual purposes of this study are to demonstrate a technique for creating a similar anisotropy in a tubular geometry appropriate to aortic replacements and to determine the structural elements responsible for anisotropy using small-angle neutron scattering.

Figure 2 demonstrates that the mechanical properties of porcine aorta can be closely matched by an anisotropic PVA conduit of similar dimensions. Indeed, an applied circumferential strain leads to the same anisotropic properties observed for samples in the form of sheets. To our knowledge this is the first polymer-based conduit that displays the anisotropic behavior of aorta. Even though our ultimate aim would be to create low diameter conduits for applications such as bypass grafts, the first step, undertaken in this study, is to demonstrate that we can develop an anisotropic conduit that specifically matches aorta (main artery). Since PVA is easily molded into any shape, applications toward smaller conduits can also be accomplished, even with different levels of anisotropy. At the same time, the effect of cyclic loading up to 10000 cycles under physiological strains $(0-30 \%)$ demonstrates that the anisotropic PVA hydrogel is an attractive material for use under these conditions.

Figure 3 shows that the mechanical properties of PVA hydrogels prepared in $\mathrm{D}_{2} \mathrm{O}$ match the anisotropic samples (all samples $75 \%$ initial strain, cycle 6) prepared using $\mathrm{H}_{2} \mathrm{O}$. This suggests that the microscopic structures of the $\mathrm{D}_{2} \mathrm{O}$ and $\mathrm{H}_{2} \mathrm{O}$ sheets are similar so that SANS data acquired on samples prepared in $\mathrm{D}_{2} \mathrm{O}$ are relevant to the microstructures of sheets prepared in $\mathrm{H}_{2} \mathrm{O}$.

The long-term mechanical behavior of the anisotropic PVA was also tested, allowing us to conclude that the stress-strain behavior of anisotropic PVA samples (75\% initial strain, cycle 6) did not change after more than 1 year after preparation. This 


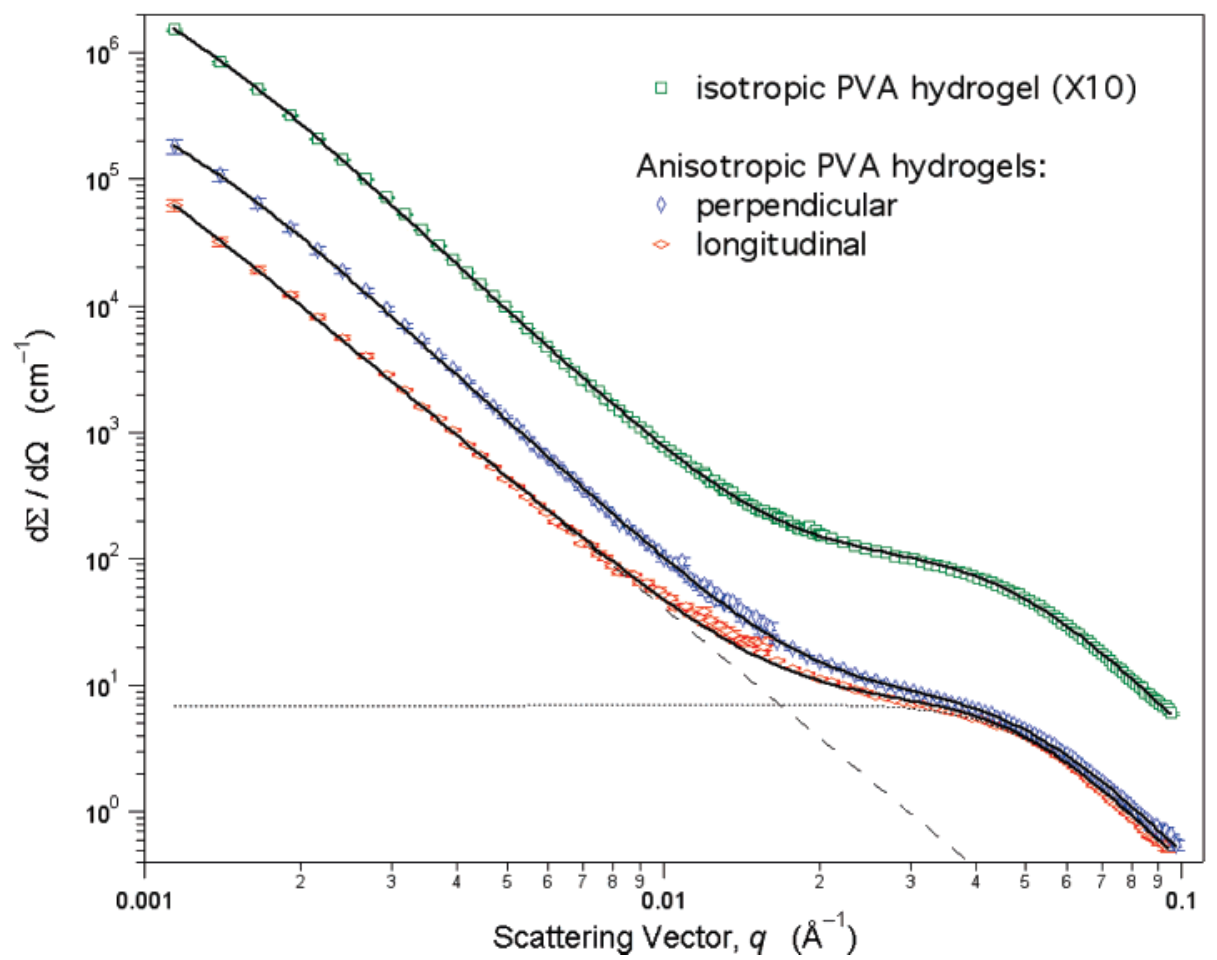

Figure 6. 1-D $\log -\log$ plots of differential scattering cross section vs scattering vector for the isotropic and both directions (longitudinal and perpendicular) of the anisotropic PVA samples. Best fits to a model based on the sum of a Beaucage and Teubner-Strey form are indicated by solid curves. The dashed and dotted curves indicate the contribution of the Beaucage and Teubner-Strey terms, respectively, for the longitudinal direction of the anisotropic sample. Note that the data and fit for the isotropic sample have been offset by a factor of 10 .

Table 1. Parameters for the Fits Indicated in Figure 6

\begin{tabular}{cccc}
\hline parameter & $\begin{array}{c}\text { isotropic } \\
\text { sample }\end{array}$ & $\begin{array}{c}\text { anisotropic } \\
\text { (longitudinal) }\end{array}$ & $\begin{array}{c}\text { anisotropic } \\
\text { (perpendicular) }\end{array}$ \\
\hline$d(\mathrm{~nm})$ & 18.7 & 17.3 & 17.5 \\
$\xi(\mathrm{nm})$ & 3.1 & 3.1 & 3.0
\end{tabular}

result implies that the PVA hydrogel has the ability to maintain its structural integrity as a function of time, which is essential for long-term implants.

Our neutron scatting data for isotropic samples support the picture developed by Kanaya ${ }^{22,23}$ using SANS and Willcox et al. ${ }^{20}$ using SAXS, TEM, and ${ }^{13} \mathrm{C}$ NMR, and recently extended by Ricciardi et al. ${ }^{25-30}$ using SANS, XRD, and solid-state ${ }^{1} \mathrm{H}$ NMR, that the PVA hydrogel is composed of two distinct phases: a somewhat homogeneous polymer-rich phase (polymer mesh) formed of swollen amorphous regions (nanopores) of size 15-30 nm cross-linked by smaller PVA crystallites, surrounding polymer-poor domains (micropores) with a characteristic length scale of $>100 \mathrm{~nm}$, which is presumably the pore structure left behind by melted ice created during the freeze-thaw process. The small value of the correlation length $\xi \sim 3 \mathrm{~nm}$ relative to the characteristic mesh length scale $(\sim 18 \mathrm{~nm})$ determined from our data indicates a low degree of order (i.e., random crystallite sites).

In the absence of a detailed model for the scattering at low $q$, we chose to fit the curve to the Beaucage form. This does not allow us to draw detailed conclusions about the large-scale structures, but some observations are clear. The length scale responsible for these features is larger than $100 \mathrm{~nm}$. The results for the anisotropic sample reveal an elongation of these structures in the direction along the applied strain. We interpret these results as indicating the presence of pores created by ice crystallization during thermal processing.

The fact that the length scales of the polymer-rich phase do not depend on induced anisotropy indicates that the nucleation and growth of polymer crystallites during thermal processing is almost independent of polymer alignment induced by the imposed strain. The lack of evidence for anisotropic structural features in the stretched sample at high $q$ indicates that the measured anisotropy in mechanical properties stems not from alignment of the crystallites but from larger features.

From the results, the following explanation of the structure is proposed. As has been suggested previously, before thermal cycling, the PVA solution is made up of a collection of random polymer chains. After the first thermal cycle, the resulting solid isotropic PVA hydrogel has a structure consisting of a weak mesh of polymer chains (amorphous phase) joined by PVA crystallites (polymer-rich phase) with a pore structure (polymerpoor phase) created by melted ice crystals. Successive thermal cycles result in ice crystals freezing and thawing mainly in the polymer poor pores, reinforcing the polymer mesh by further crystallization through growth of primary crystallites as well as secondary crystallites..$^{20,30}$ Figure 7 shows the proposed schematic of the effect of freeze/thaw cycling on the structure of isotropic PVA. Notice the structural dimension of about 3 $\mathrm{nm}$ (crystallites), with average distance in between crystallites of about $19 \mathrm{~nm}$. Polymer-poor regions (macropores) are larger than $100 \mathrm{~nm}$.

Our anisotropic PVA samples are prepared by applying a strain to an isotropic (cycle 1) sample and subjecting it to additional thermal cycles. We suggest that when the cycle 1 sample is stretched, the polymer mesh (crystallites embedded in an amorphous phase) and polymer-poor phase (larger pores) elongate along the stretching direction while the crystallite distribution remains largely random. Successive thermal cycles result in ice crystals freezing and thawing predominantly in the same elongated pores, reinforcing the polymer mesh by further crystallization. The semioriented primary crystallites will grow in size, but other randomly oriented secondary crystallites will also be formed, ${ }^{20,30}$ producing additional cross-links which 
Cycle 0

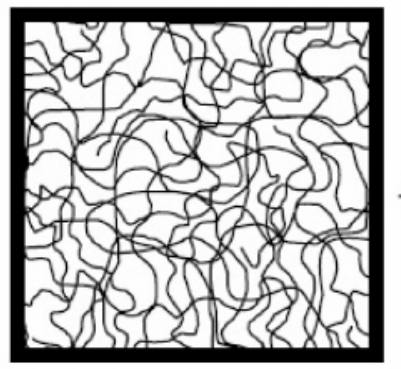

Cycle 1

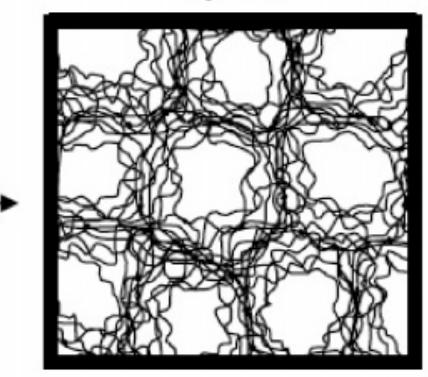

Cycle 6

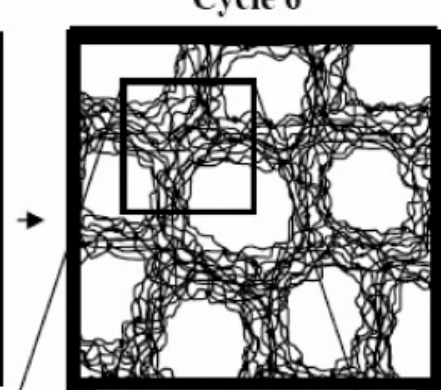

Crystallites
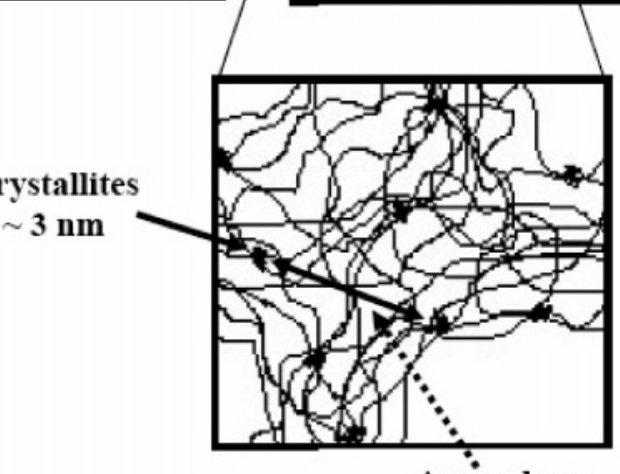

Amorphous

$\sim 19 \mathrm{~nm}$

Figure 7. Proposed schematic of the effect of freeze/thaw cycling on the structure of isotropic PVA. Notice the structural dimensions of about 3 $\mathrm{nm}$ (crystallites), with average distance in between crystallites of about $19 \mathrm{~nm}$.

\section{Isotropic Thermal Cycling}

Cycle 1

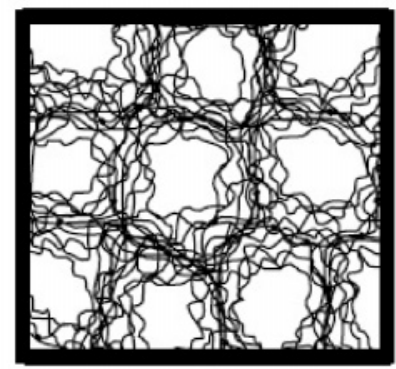

$\$ Cycle 1 Sample Stretched
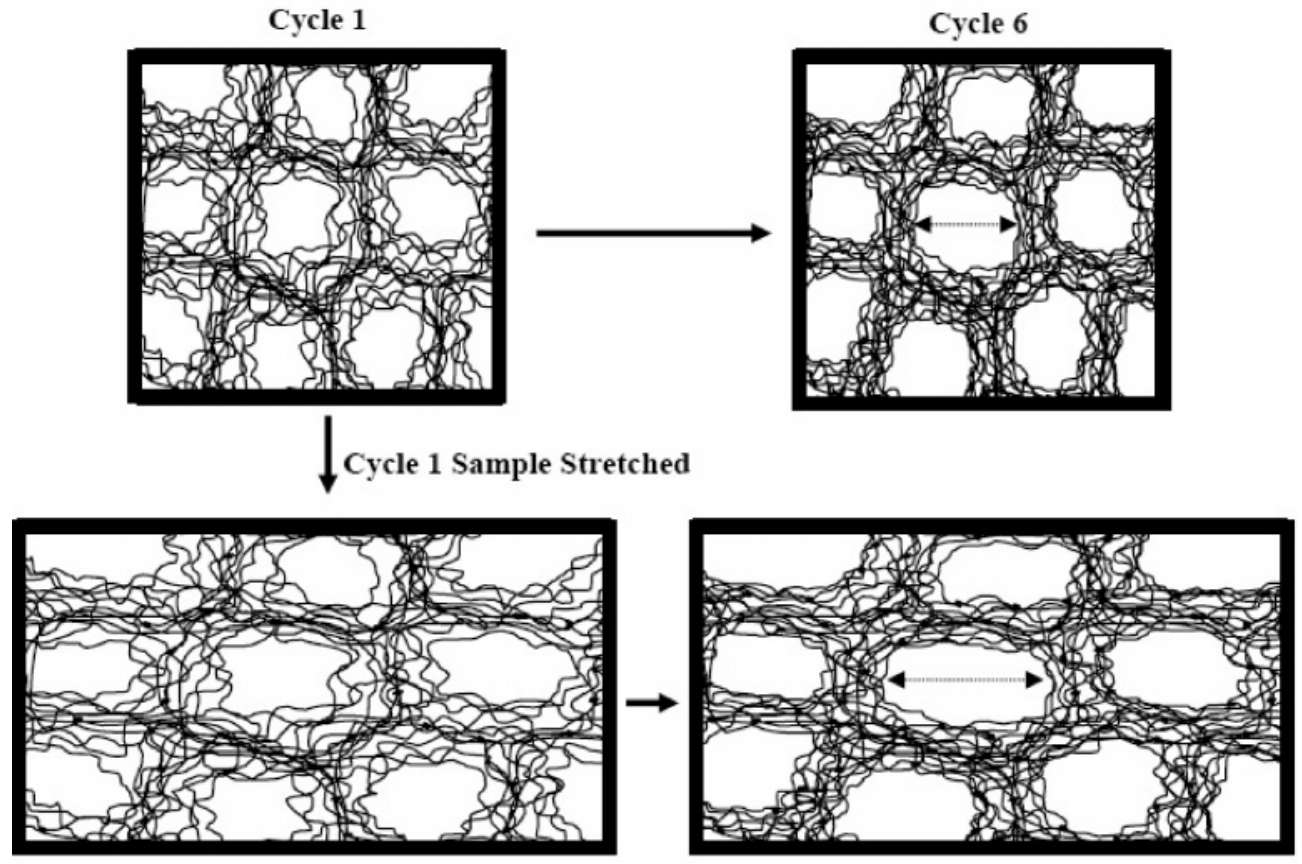

Anisotropic Thermal Cycling

Figure 8. Proposed schematic of the freeze/thaw cycling under an applied constant initial strain for the creation of the anisotropic PVA structure. Notice the elongated (longer arrows) macropores for anisotropic PVA.

increase the mechanical strength. When the thermal processing is complete and the imposed strain removed, a sample with permanently elongated large pores (polymer-poor phase) and anisotropic properties remains. This model is also consistent with the results reported previously, where the ratio of stress in the longitudinal to the perpendicular direction (at constant strain) remained independent of the number of thermal cycles and was only affected by the amount of initial strain applied. The increase in the number and size of randomly oriented crystallites with increasing thermal cycles only serves to strengthen the PVA matrix as a whole but does not affect the degree of anisotropy. An increase in initial strain, however, increases the elongation of the larger pores, resulting in an increase in the degree of anisotropy. ${ }^{18}$ Figure 8 shows the proposed schematic of the freeze/thaw cycling under an applied constant initial strain for the creation of the anisotropic PVA structure. Notice the 
elongated polymer-poor pores (surrounded by polymer mesh) for the anisotropic scheme.

Ultrasmall-angle neutron scattering (USANS), which probes smaller values of $q$ (larger structures), combined with improved modeling motivated by these preliminary results, are being conducted for anisotropic PVA hydrogels under different conditions (e.g., different initial strains and numbers of thermal cycles). This will provide a better understanding of the structure of the PVA hydrogel and its anisotropy.

\section{Conclusion}

We have created an anisotropic PVA conduit that closely matches the tensile properties of porcine aorta past the physiological range in both the circumferential and the axial directions. Characterization of anisotropic $\mathrm{PVA}-\mathrm{D}_{2} \mathrm{O}$ hydrogels by neutron scattering reveals that the anisotropic mechanical properties are due to the large-scale pore structure of the gel and not the structure of the PVA crystallites themselves, which serve only as physical cross-linkers. Depending on the application, specific composition, processing parameters, and amount of initial strain can be chosen to create an isotropic or anisotropic PVA biomaterial with mechanical properties similar to that of the tissue to be replaced.

Acknowledgment. The authors thank the Natural Science and Engineering Research Council of Canada (NSERC), the Advanced Foods and Materials Network (AFMNet) and the Ontario Graduate Scholarship (OGS) program for financial support. We are also grateful to the University Machine Shop for fabrication of sample molds and accessories. This work utilized facilities supported in part by the National Science Foundation under Agreement DMR-0454672. We acknowledge the support of the National Institute of Standards and Technology (NIST), U.S. Department of Commerce, in providing the neutron research facilities used in this work.

\section{References and Notes}

(1) Thomas, A. C.; Campbell, G. R.; Campbell, J. H. Cardiovasc. Pathol. 2003, 12, 271-276.

(2) Popma, J. J.; Sawyer, M.; Selwyn, A. P.; Kinlay, S. Am. J. Cardiol. 2000, $86,18 \mathrm{H}-28 \mathrm{H}$.

(3) Moustapha, A.; Anderson, H. V. Curr. Opin. Cardiol. 2000, 15, 46371.

(4) Rashid, S. T.; Salacinski, H. J.; Fuller, B. J.; Hamilton, G.; Seifalian, A. M. Cell Proliferation 2004, 37, 351-366.

(5) Haruguchi, H.; Teraoka, S. J. Artif. Organs 2003, 6, 227-35.

(6) Xue, L.; Greisler, H. P. J. Vasc. Surg. 2003, 37, 472-80.

(7) Schoen, F. J. J. Heart Valve Disease 1999, 8, 350-8.

(8) Fung, Y. C. Biomechanics: Mechanical Properties of Living Tissues, 2nd ed.; Springer-Verlag: New York, 1993; p 321.
(9) Hayashi, K.; Stergiopulos, N.; Meister, J.; Greenwald, S. E.; Rachev, A. In Biomechanical Techniques: Techniques and Applications; Leondes, C., Ed.; CRC Press: Boca Raton, FL, 2001; Vol. 2, p 2.

(10) Abe, H., Hayashi, K., Sato, M., Eds. Data Book on Mechanical Properties of Living Cells, Tissues, and Organs; Springer-Verlag: Tokyo, 1996.

(11) Paradossi, G.; Cavalieri, F.; Chiessi, E.; Spagnoli, C.; Cowman, M. K. J. Mater. Sci.: Mater. Med. 2003, 14, 687-691.

(12) Mori, Y.; Tokura, H.; Yoshikawa, M. J. Mater. Sci. 1997, 32, 491496.

(13) Hassan, C. M.; Peppas, N. A. Adv. Polym. Sci. 2000, 153, 37-65.

(14) Lozinsky, V. I.; Plieva, F. M. Enzyme Microb. Technol. 1998, 23 227-242.

(15) Wan, W. K.; Campbell, G.; Zhang, Z. F.; Hui, A. J.; Boughner, D. R. J. Biomed. Mater. Res. 2002, 63, 854-861.

(16) Stammen, J. A.; Williams, S.; Ku, D. N.; Guldberg, R. E. Biomaterials 2001, 22, 799-806

(17) Millon, L. E.; Wan, W. K. J. Biomed. Mater. Res., Part B: Appl. Biomater. 2006, 79, 245-53.

(18) Millon, L. E.; Mohammadi, H.; Wan, W. K. J. Biomed. Mater. Res., Part B: Appl. Biomater. 2006, 79, 305-11.

(19) Fergg, F.; Keil, F. J.; Quader, H. Colloid Polym. Sci. 2001, 279, 6167.

(20) Willcox, P. J.; Howie, D. W.; Schmidt-Rohr, K.; Hoagland, D. A.; Gido, S. P.; Pudjijanto, S.; Kleiner, L. W.; Venkatraman, S. J. Polym. Sci., Part B: Polym. Phys. 1999, 37, 3438-3454.

(21) Takeshita, H.; Kanaya, T.; Nishida, K.; Kaji, K. Macromolecules 1999, 32, 7815-7819.

(22) Kanaya, T.; Ohkura, M.; Kaji, K.; Furusaka, M.; Misawa, M. Macromolecules 1994, 27, 5609-15.

(23) Kanaya, T.; Ohkura, M.; Takeshita, H.; Kaji, K.; Furusaka, M.; Yamaoka, H.; Wignall, G. D. Macromolecules 1995, 28, 3168-74.

(24) Takeshita, H.; Kanaya, T.; Nishida, K.; Kaji, K.; Takahashi, T.; Hashimoto, M. Phys. Rev. E 2000, 61, 2125-2128.

(25) Ricciardi, R.; Gaillet, C.; Ducouret, G.; Lafuma, F.; Laupretre, F. Polymer 2003, 44, 3375-3380.

(26) Ricciardi, R.; Auriemma, F.; Gaillet, C.; De Rosa, C.; Laupretre, F. Macromolecules 2004, 37, 9510-9516.

(27) Ricciardi, R.; Auriemma, F.; De Rosa, C.; Laupretre, F. Macromolecules 2004, 37, 1921-1927.

(28) Ricciardi, R.; Auriemma, F.; De Rosa, C. Macromol. Symp. 2005, 222, 49-63.

(29) Ricciardi, R.; D’Errico, G.; Auriemma, F.; Ducouret, G.; Tedeschi, A. M.; De Rosa, C.; Laupretre, F.; Lafuma, F. Macromolecules 2005 , 38, 6629-6639.

(30) Ricciardi, R.; Mangiapia, G.; Lo, Celso, F.; Paduano, L.; Triolo, R.; Auriemma, F.; De Rosa, C.; Laupretre, F. Chem. Mater. 2005, 17, 1183-1189.

(31) Mayne, A. S.; Christie, G. W.; Smaill, B. H.; Hunter, P. J.; BarrattBoyes, B. G. J. Thoracic Cardiovasc. Surg. 1989, 98, 170-80.

(32) Hansen, B.; Menkis, A. H.; Vesely, I. Ann. Thoracic Surg. 1995, 60, S384-90.

(33) Higgins, J. S.; Benoit, H. C. Polymers and Neutron Scattering; Clarendon Press: Oxford, 1994.

(34) Teubner, M.; Strey, R. J. Chem. Phys. 1987, 87, 3195-200.

(35) Beaucage, G. J. Appl. Crystallogr. 1995, 28, 717-728.

MA062781F 